\title{
Estimación de la repetitividad y selección genética de árboles de cacao aromático con material genético de EE-INIA-San Martin y de la UC de Lebuaf, en Perú
}

\author{
Estimation of repeatability and genetic selection of aromatic cacao trees \\ with genetic material from EE-INIA-San Martin and UC Lebuaf in Peru \\ Carlos Oliva ${ }^{1, *}$, José Benito ${ }^{2}$, Ronald Acuña ${ }^{3}$, Ana Bocanegra ${ }^{3}$, Jhordan Baltazar ${ }^{3}$ \\ Phi Peruvian Consultores SAC. Calle Las Alondras \#194, Callao, Lima, Perú. \\ Estación Experimental INIA- Jr. Martínez de Compagñón \#1035, San Martín, Tarapoto, Perú. \\ LEBUAF SAC. Fundo Plantación María s/n, San Martín, Huimbayoc, Perú.
}

Recibido 21 agosto 2013. Aceptado 20 marzo 2014.

\begin{abstract}
Resumen
El cultivo de cacao, es una de las actividades más importantes en la Amazonía Peruana. Su base genética está sustentada en la introducción de clones mejorados, por lo que el potencial genético local que están en los bancos de germoplasma no está siendo bien utilizados debido al limitado estudio en mejoramiento genético. Este trabajo tuvo por objetivo estimar la repetibilidad para la selección genética de árboles con alto rendimiento de granos de cacao aromático. El análisis de selección genética fue ejecutado con el software SELEGEN REML/BLUP, en una data de 3 años de evaluación de rendimiento de granos kg/árbol, peso promedio de granos $(\mathrm{g})$ y peso promedio de mazorcas $(\mathrm{kg})$ en el banco de Germoplasma de INIA-San Martin y Unidad de Conservación de Lebuaf SAC. La repetibilidad individual para rendimiento de granos/árbol y peso promedio de granos fue moderada con $(0,52)$ y $(0,30)$ respectivamente, lo que no ocurrió con el peso promedio de la mazorca que fue baja con $(0,24)$. La variable con mayor estabilidad fue el rendimiento de granos/árbol, quien arrojo en 3 cosechas 0,77 , propiciando una exactitud selectiva de 0,88 . Genotipos superiores pueden ser seleccionados con precisión con 4 a 5 cosechas. La selección y clonación de los 20 mejores genotipos o árboles selectos deberán propiciar una ganancia genética de 112\%, elevando la productividad media anual de granos por árbol de 1,24 kg/árbol a 1,54kg/árbol.
\end{abstract}

Palabras clave: SELEGEN, fenotipos, genotipos, árbol, repetitividad, exactitud y cacao.

\begin{abstract}
Cocoa farming is one of the most important activities in the Peruvian Amazon. Its genetic basis is supported by the introduction of improved clones, so that the local genetic potential are in germplasm bank is not well used due to limited breeding study. This study aimed to estimate the repeatability for the genetic selection of high-yield trees with aromatic cocoa beans. Genetic selection analysis was executed with the SOFTWARE SELEGEN REML / BLUP in a 3-year data for assessing grain yield $\mathrm{kg} /$ tree, average grain weight (g) and average weight of cobs $(\mathrm{kg})$ in the bank germplasm INIA-San Martin and Lebuaf Conservation Unit SAC (Plantation Maria). The individual repeatability grain yield / tree and average grain weight was moderate $(0.52)$ and $(0.30)$ respectively, which did not happen for the repeatability of the variable average cob weight was low with (0.24). The variable was more stable grain yield / tree, who threw an average of 3 harvest repeatability was 0.77 , favoring selective accuracy of 0.88 . Superior genotypes can be accurately selected with 4 to 5 harvests. The selection and cloning of the 20 best genotypes or selected trees should facilitate a genetic gain of $112 \%$, raising the annual average productivity of 1.24 grains per tree $\mathrm{kg} /$ tree to $1.54 \mathrm{~kg} /$ tree.
\end{abstract}

Keywords: SELEGEN, tree, repeatability, accuracy and cocoa.

\footnotetext{
* Autor para correspondencia

E-mail: olivaproyectos@gmail.com (C. Oliva)
} 


\section{Introducción}

En el Perú se estima que existen al orden de 68650 ha de cacao, distribuido en más de 10 regiones, de las cuales en San Martin se registra al menos 18 mil ha en diferentes etapas de crecimiento y desarrollo (García, 2010).

Se estima que entre el Banco de Germoplasma de INIA y la Unidad de Conservación (UC) de la empresa Lebuaf, se tiene aproximadamente 28 clones, siendo el de mayor distribución en las plantaciones de la Amazonía Peruana, el clon CCN51 con al menos el 80\% (Benito, 2010).

Hay clones que ingresaron a nuestro país, con alta calidad genética, pero a través de los años esto se ha ido degenerando de generación en generación, debido al mal uso del recurso como fuente de semilla que no ha controlado la pureza y calidad. Sobre esta base y conociendo la amplia disponibilidad de material genético y su alto potencial que están en el Banco de Germoplasma de UNIA y la UC de Lebuaf, se considera fundamental, dar inicio a trabajos de selección genética, mediante herramientas modernas como es el SELEGEN RELM/BLUP, que podría ayudarnos a seleccionar clones de alto rendimiento sobre la cual se pueda consolidad a futuro el trabajo de mejoramiento genético del cacao. Pero paralelo a ello, con la confiabilidad de la selección genética, es posible promover la clonación e instalar huertos semilleros para la provisión de semillas con alto nivel de ganancia genética (De Resende y De Oliveira, 1997).

La propagación vegetativa representa una alternativa valiosa para la multiplicación masiva de genotipos superiores, sin depender de las variaciones típicas asociadas a la producción de semilla. Además, al contrario de la reproducción por semilla, que aprovecha sólo la porción aditiva de la varianza genética, la propagación vegetativa permite doble ganancia genética convirtiéndolo en una de las alternativas más atractivas en mejoramiento para el incremento de la productividad (Leakey y Mesén, 1991).

En base a ello, en el presente trabajo se utilizó el material genético disponible y sobre la cual se realizó una selección genética de cacao aromático, con el programa de selección genética computarizada.

\section{Material y métodos}

\section{Material genético}

El material genético utilizado en las evaluaciones de rendimiento de cacao, está distribuido en al menos 15 clones (ICS-95, HIBRIDO, RONALD, CCN-51, CRIOLLO, ICS-1, P-7 (PAO7), IMC-67, ICS-6, P-12 (PAO12), ICS-39, CCS1, CCS-2, CCS-3 y CCS-4), los mismos que están instalados en las plantaciones de la Empresa LEBUAF y en el Banco de Germoplasma de INIA-San Martin. Las plantas están instaladas en densidades similares $3,0 \mathrm{~m} \times 3,0 \mathrm{~m}(3 \times 3 \mathrm{~m})$ y con manejos agronómicos parecidos, condición que ayuda a homogeneizar variables que facilitará evitar sesgos en la obtención de los resultados.

\section{Repetibilidad de la producción de frutos}

Se sistematizó la data de 3 años de evaluación de rendimiento de frutos en 100 árboles seleccionados entre la Empresa LEBUAF y el Banco de Germoplasma de INIA. Estas plantas están ubicadas en suelos de textura franco arcilloso orden Entisols y Sub orden Fluvents, con $\mathrm{pH}$ cambiando de 6,4 a 7,6. El clima se caracteriza por ser cálido y húmedo con una temperatura media anual de 25,1 y rango de 17,4 a 36,5 grados Celsius. La precipitación media anual es de 1447,31 mm. La matriz de datos de tres cosechas consecutivas (2010 al 2012) fue analizada en programa genético computarizado Selegen-Reml/Blup (De Resende, 2002), permitiendo conocer la repetibilidad, exactitud selectiva ganancia genética con la selección de los caracteres de producción. 
Los valores genotípicos $(\hat{g})$ de cada planta serán estimados por:

$\hat{g}=M G+B_{p}\left(M P_{j}-M G\right)$

Donde $M G$ : media general de las plantas en varias mediciones; $B_{p}$ : coeficiente de determinación del valor fenotípico permanente $=(m * \hat{p}) /[1+(m-1) * \hat{p}]$; $m$ : media de 4 mediciones en el individuo; $\hat{p}$ : estimativa de la repetibilidad individual; $M P_{j}$ : media general da la planta $j$ en las varias mediciones.

La ganancia genética será estimada como el promedio de valores genotípicos de los individuos seleccionados.

\section{Resultados y discusión}

La repetitividad individual para kilos de granos/árbol, peso promedio de granos y peso promedio de mazorcas son de 0,52; 0,30 y 0,24 , respectivamente. $\mathrm{La}$ repetitividad del promedio de 3 cosechas para estas mismas variables es 0,$77 ; 0,56 \mathrm{y}$ 0,49 (Tabla 1).

\section{Tabla 1}

Componentes de varianza asociados a la producción en Material Genético de Cacao de INIA-San Martin y Lebuaf SAC, estimados por máxima verosimilitud restringida (REML)

\begin{tabular}{|c|c|c|c|}
\hline $\begin{array}{c}\text { Compo- } \\
\text { nentes }\end{array}$ & $\begin{array}{c}\text { Rendimiento } \\
\text { masa de } \\
\text { grano/planta } \\
(\mathrm{kg})\end{array}$ & $\begin{array}{c}\text { Masa } \\
\text { promedio } \\
\text { granos } \\
(\mathrm{g})\end{array}$ & $\begin{array}{c}\text { Masa } \\
\text { promedio } \\
\text { mazorca } \\
(\mathrm{kg})\end{array}$ \\
\hline$V f p^{(i)}$ & 0,045 & 0,041 & 0,007 \\
\hline$V e t^{(\mathrm{ii})}$ & 0,041 & 0,096 & 0,022 \\
\hline$V f^{(\text {iii) }}$ & 0,086 & 0,137 & 0,028 \\
\hline$R^{\text {(iv) }}$ & $\begin{array}{c}0,52 \\
\pm 0,1191\end{array}$ & $\begin{array}{c}0,299 \\
\pm 0,0899\end{array}$ & $\begin{array}{c}0,239 \\
\pm 0,0804\end{array}$ \\
\hline$r m^{(\mathrm{v})}$ & 0,769 & 0,561 & 0,486 \\
\hline$A c m^{(v i)}$ & 0,877 & 0,749 & 0,697 \\
\hline$M G$ & 1,258 & 1,732 & 0,510 \\
\hline
\end{tabular}

(i) Varianza fenotípica permanente

(ii) Varianza ambiental temporaria

(iii) Varianza fenotípica individual

(iv) Repetibilidad individual

(v) Repetibilidad de media de cosechas

${ }^{\text {(vi) }}$ Exactitud selectiva.
Así, la selección basada en este promedio propicia exactitud selectiva de 0,88 para la variable peso de granos/árbol, 0,75 para peso promedio de granos y 0,70 para peso promedio de mazorca. Con estos indicadores es posible realizar la selección de genotipos superiores con alto nivel de precisión.

La repetibilidad se refiere a la correlación entre medidas repetidas realizadas en un mismo individuo. La magnitud observada para la variable rendimiento granos/árbol es 0,52 en este trabajo es considerada moderado, para la variable peso promedio de granos fue de 0,30 también considerada moderada y para la variable peso promedio de mazorcas de la repetibilidad fue 0,24 considerada baja, según escala de categorización de la repetibilidad definido por (De Resende y De Oliveira, 1997; De Resende, 2002).

Coeficiente de selección, exactitud selectiva y eficiencia del uso de " $m$ " mediciones para un buen programa de mejoramiento

Rendimiento (granos / arbol de cacao)

Deseando una exactitud del $90 \%$ en la selección, o sea, $82 \%$ de determinación, indica que se deben evaluar 4 cosechas por planta (m) (Tabla 2).

\section{Tabla 2}

Coeficiente de determinación, exactitud selectiva y eficiencia del uso de m mediciones repetidas en vez de una, asociados a la producción de granos de cacao

\begin{tabular}{cccc}
\hline $\mathrm{m}$ & Determinación & Precisión & Eficiencia \\
\hline 1 & 0,53 & 0,73 & 1,00 \\
2 & 0,69 & 0,83 & 1,14 \\
3 & 0,77 & 0,88 & 1,21 \\
$\mathbf{4}$ & $\mathbf{0 , 8 2}$ & $\mathbf{0 , 9 0}$ & $\mathbf{1 , 2 5}$ \\
5 & 0,85 & 0,92 & 1,27 \\
6 & 0,87 & 0,93 & 1,29 \\
7 & 0,89 & 0,94 & 1,30 \\
8 & 0,90 & 0,95 & 1,31 \\
9 & 0,91 & 0,95 & 1,31 \\
10 & 0,92 & 0,96 & 1,32 \\
\hline
\end{tabular}


Esto propicia una confiabilidad selectiva del $90 \%$, la cual es adecuada para el propósito del mejoramiento genético. Propicia también una eficiencia de 1,25 (superioridad de $0,25 \%$ ) con relación al uso de solo una cosecha.

Peso promedio de grano de cacao aromático

Estimando una exactitud del $83 \%$ en la selección, o sea, $68 \%$ de determinación, indica que se deben evaluar 5 cosechas por planta o árbol (Tabla 3). Esto propicia una confiabilidad selectiva del $83 \%$, la cual es adecuada para el propósito del mejoramiento genético del cacao. Se encuentra una eficiencia de 1,51 (superioridad de $0,51 \%$ ) con relación al uso de solo una cosecha. Se observa que esta variable es menos consistente que los valores obtenidos en la variable rendimiento granos $\mathrm{kg} /$ árbol, lo que hace indicar que existe mayor proporción del efecto del medio ambiente.

\section{Tabla 3}

Coeficiente de determinación, exactitud selectiva y eficiencia del uso de $\mathrm{m}$ mediciones repetidas en vez de una, asociados al peso promedio de granos de cacao

\begin{tabular}{cccc}
\hline $\mathrm{m}$ & Determinación & Precisión & Eficiencia \\
\hline 1 & 0,30 & 0,55 & 1,00 \\
2 & 0,46 & 0,68 & 1,24 \\
3 & 0,56 & 0,75 & 1,37 \\
4 & 0,63 & 0,79 & 1,45 \\
$\mathbf{5}$ & $\mathbf{0 , 6 8}$ & $\mathbf{0 , 8 3}$ & $\mathbf{1 , 5 1}$ \\
6 & 0,72 & 0,85 & 1,55 \\
7 & 0,75 & 0,87 & 1,58 \\
8 & 0,77 & 0,88 & 1,61 \\
9 & 0,79 & 0,89 & 1,63 \\
10 & 0,81 & 0,90 & 1,65 \\
\hline
\end{tabular}

\section{Peso de mazorca de cacao aromatico}

Estimando una exactitud del $78 \%$ en la selección, o sea, $61 \%$ de determinación, indica que se deben evaluar 5 cosechas por planta o árbol (Tabla 4). Esto propicia una confiabilidad selectiva del 78, la cual regularmente adecuada para el propósito del mejoramiento genético del cacao es decir dispone de cierto nivel de inexactitud. Se encuentra una eficiencia de 1,60 (superioridad de 0,60\%) con relación al uso de solo una cosecha. El comportamiento de esta última variable podría explicar la amplia variabilidad en relación al peso del fruto, por otro lado también explica la importancia del medio ambiente que influye drásticamente en el peso de la mazorca, por esta razón la repetibilidad reportada es baja, la cual implica que encima del $22 \%$ de sebe a efecto ambientales.

\section{Tabla 4}

Coeficiente de determinación, exactitud selectiva y eficiencia del uso de $\mathrm{m}$ mediciones repetidas en vez de una, asociados al peso promedio de mazorca de cacao

\begin{tabular}{cccc}
\hline $\mathrm{m}$ & Determinación & Precisión & Eficiencia \\
\hline 1 & 0,24 & 0,49 & 1,00 \\
2 & 0,39 & 0,62 & 1,27 \\
3 & 0,49 & 0,70 & 1,42 \\
4 & 0,56 & 0,75 & 1,53 \\
5 & $\mathbf{0 , 6 1}$ & $\mathbf{0 , 7 8}$ & $\mathbf{1 , 6 0}$ \\
6 & 0,65 & 0,81 & 1,65 \\
7 & 0,69 & 0,83 & 1,69 \\
8 & 0,72 & 0,85 & 1,73 \\
9 & 0,74 & 0,86 & 1,76 \\
10 & 0,76 & 0,87 & 1,78 \\
\hline
\end{tabular}

Selección de árboles plus de cacao aromático

Los resultados de la selección genética con base en TRES cosechas revelan un alto potencial para obtener ganancia genética en esta población. La selección y clonación de los 20 mejores individuos deberá propiciar una ganancia genética de $112 \%$, elevando la productividad media anual por árbol de $1,26 \mathrm{~kg}$ grano/año para $1,54 \mathrm{~kg}$ grano/año (Tabla 5). Esto es debido al hecho de que la especie demuestra niveles de variabilidad en la data de selección está involucrado 15 clones, con amplia variabilidad genética disponible para selección y mejoramiento. Así, programas de mejoramiento genético de cacao para nuestro país son muy estratégicos y esenciales para el desarrollo del sector productivo asociado a esta especie. 


\section{Tabla 5}

Selección genética asociados a la producción de granos de cacao en arboles de cacao en el Banco de Germoplasma de Cacao INIA - San Martin y Unidad de Conservación de la Empresa LEBUAF SAC. - Perú, con base en TRES cosechas

\begin{tabular}{ccccccc}
\hline Orden & Árbol & Valor Fenotípico & $\begin{array}{c}\text { Valor } \\
\text { Genotípico }\end{array}$ & $\begin{array}{c}\text { Ganancia } \\
\text { Genética }\end{array}$ & $\begin{array}{c}\text { Promedio } \\
\text { Población } \\
\text { Mejorada }\end{array}$ & Ganancia (\%) \\
\hline 1 & $\mathbf{7 4}$ & 0,61 & 1,86 & 0,61 & 1,86 & 149,1 \\
2 & $\mathbf{7 6}$ & 0,48 & 1,74 & 0,54 & 1,80 & 139,1 \\
3 & $\mathbf{1 7}$ & 0,39 & 1,65 & 0,49 & 1,75 & 131,7 \\
4 & $\mathbf{9 3}$ & 0,37 & 1,63 & 0,46 & 1,72 & 130,5 \\
5 & $\mathbf{7 5}$ & 0,37 & 1,62 & 0,44 & 1,70 & 129,8 \\
6 & $\mathbf{5 2}$ & 0,36 & 1,61 & 0,43 & 1,69 & 129,0 \\
7 & $\mathbf{6 8}$ & 0,33 & 1,59 & 0,41 & 1,67 & 127,0 \\
8 & $\mathbf{7 2}$ & 0,31 & 1,56 & 0,40 & 1,66 & 125,1 \\
9 & $\mathbf{9 2}$ & 0,31 & 1,56 & 0,39 & 1,65 & 125,1 \\
10 & $\mathbf{9}$ & 0,30 & 1,56 & 0,38 & 1,64 & 124,7 \\
11 & $\mathbf{6 6}$ & 0,27 & 1,53 & 0,37 & 1,63 & 122,2 \\
12 & $\mathbf{6 0}$ & 0,22 & 1,48 & 0,36 & 1,62 & 118,2 \\
13 & $\mathbf{7 1}$ & 0,22 & 1,48 & 0,35 & 1,61 & 118,2 \\
14 & $\mathbf{2 3}$ & 0,22 & 1,47 & 0,34 & 1,60 & 117,9 \\
15 & $\mathbf{4 2}$ & 0,19 & 1,45 & 0,33 & 1,59 & 115,9 \\
16 & $\mathbf{9 6}$ & 0,17 & 1,43 & 0,32 & 1,58 & 114,5 \\
17 & $\mathbf{7 3}$ & 0,16 & 1,41 & 0,31 & 1,57 & 113,0 \\
18 & $\mathbf{8 9}$ & 0,15 & 1,41 & 0,30 & 1,56 & 112,4 \\
19 & $\mathbf{3 4}$ & 0,14 & 1,40 & 0,29 & 1,55 & 112,0 \\
20 & $\mathbf{8}$ & 0,13 & 1,40 & 0,28 & 1,54 & 112,0 \\
\hline
\end{tabular}

Ganancias mayores podrían obtenerse con la selección de los cinco mejores árboles, pero un número muy pequeño de árboles madres podría causar vulnerabilidad de los plantíos comerciales oriundos de ellas. Eso es debido a la baja variabilidad genética asociada a un pequeño tamaño efectivo poblacional.

Sobre la base de esta selección de árboles es posible proceder a la instalación de Jardin Clonal para la disponibilidad de semilla vegetativa y sobre ello promover ampliar la frontera agrícola del cacao proyectando mejores rendimiento en base al rendimiento promedio nacional actual que esta al orden del 0,7 t/ha, es decir un rendimiento de 0,6 kg grano/árbol.

\section{Conclusiones}

La repetibilidad de la producción fue de moderada magnitud, mostrando que la heredabilidad de carácter es moderada. Para trabajos futuros se estima seleccionar con base en cuatro evaluaciones por árbol. Se ha logrado la selección de los 20 mejores árboles, cuya ganancia genética futura equivale al $112 \%$, en comparación al rendimiento promedio actual de granos que es al orden de 1,24 kg grano/árbol. La selección y clonación de los diez mejores individuos deberá propiciar una ganancia genética importante, elevando la productovidad media anual de frutas por planta. Se recomienda utilizar los 10 mejores árboles para la instalación de un Jardín Clonal. 


\section{Referencias bibliográficas}

Benito, J. 2010. Resultados de investigación de 21 clones internacionales. EEA.Tulumayo - Tingo María Perú.1980-1987.

De Resende, M.D.V.; de Oliveira, E.B. 1997. Sistema "SELEGEN" - Seleção Genética Computadorizada para o Melhoramento de Espécies Perenes. Pesquisa Agropecuária Brasileira 32(9): 931-939.

De Resende, M.D.V. 2002. Software SELEGEN REML/BLUP. EMBRAPA, Colombo. Manual Selegen.
García, C.L. 2010. Catálogo de Cultivares de Cacao del Perú. Ministerio de Agricultura- DEVIDA. LimaPerú. 111 pag.

Leakey, B.; Mesén, F. 1991. Métodos de propagación vegetativa en árboles tropicales: enraizamiento de estacas suculentas. Capítulo 10. In: Manual sobre Mejoramiento Genético Forestal con Referencia Especial a América Central. Cornelius, J.P.; Mesén, F.; Corea, E. (eds.). Proyecto Mejoramiento Genético Forestal, CATIE, Turrialba, Costa Rica: 135-153. 